\title{
Isovector and flavor diagonal charges of the nucleon from 2+1+1 flavor QCD
}

\author{
Rajan Gupta*, Tanmoy Bhattacharya, Vincenzo Cirigliano, Boram Yoon \\ Theoretical Division T-2, Los Alamos National Laboratory, Los Alamos, NM 87545, USA \\ E-mail: rgelanl.gov, ciriglianodlanl.gov, tanmoyelanl.gov, \\ boramelanl.gov
}

\section{Yong-Chull Jang}

Phsics Department, Brookhaven National Laboratory, Upton, NY 11973, USA

E-mail: ypjabnl.gov

\section{Huey-Wen Lin}

Department of Physics and Astronomy \& Computational Mathematics, Science and Engineering Michigan State University, East Lansing, MI 48824 E-mail: hwl in @ pa.msu . edu

\section{PNDME Collaboration}

\begin{abstract}
We present high-statistics results for the isovector and flavor diagonal charges of the proton using 11 ensembles of $2+1+1$ flavor HISQ fermions. In the isospin symmetric limit, results for the neutron are given by the $u \leftrightarrow d$ interchange. A chiral-continuum fit with leading order corrections was made to extract the connected and disconnected contributions in the continuum limit and at $M_{\pi}=135 \mathrm{MeV}$. All results are given in the $\overline{M S}$ scheme at $2 \mathrm{GeV}$. The isovector charges, $g_{A}^{u-d}=1.218(25)(30), g_{S}^{u-d}=1.022(80)(60)$ and $g_{T}^{u-d}=0.989(32)(10)$, are used to obtain low-energy constraints on novel scalar and tensor interactions, $\varepsilon_{S}$ and $\varepsilon_{T}$, at the TeV scale. The flavor diagonal axial charges are: $g_{A}^{u} \equiv \Delta u \equiv\langle 1\rangle_{\Delta u^{+}}=0.777(25)(30)$, $g_{A}^{d} \equiv \Delta d \equiv\langle 1\rangle_{\Delta d^{+}}=-0.438(18)(30)$, and $g_{A}^{s} \equiv \Delta s \equiv\langle 1\rangle_{\Delta s^{+}}=-0.053(8)$. Their sum gives the total quark contribution to the proton spin, $\sum_{q=u, d, s}\left(\frac{1}{2} \Delta q\right)=0.143(31)(36)$. This result is in good agreement with the recent COMPASS analysis $0.13<\frac{1}{2} \Delta \Sigma<0.18$. Implications of results for the flavor diagonal tensor charges, $g_{T}^{u}=0.784(28)(10), g_{T}^{d}=-0.204(11)(10)$ and $g_{T}^{s}=-0.0027(16)$ for constraining the quark electric dipole moments and their contributions to the neutron electric dipole moment are discussed. These flavor diagonal charges also give the strength of the interaction of dark matter with nucleons via axial and tensor mediators.
\end{abstract}

The 36th Annual International Symposium on Lattice Field Theory - LATTICE2018

22-28 July, 2018

Michigan State University, East Lansing, Michigan, USA.

\footnotetext{
* Speaker.
} 


\section{Introduction}

Lattice QCD provides first principal results for the matrix elements (ME) of quark bilinear operators within nucleon states that are needed to quantify a number of properties and structure of the nucleon. All the calculations reported on here have been done using clover valence fermions on 2+1+1 flavor HISQ ensembles generated by the MILC collaboration [1]. Detailed analyses and results, summarized here, for the isovector charges are given in Ref. [2], for flavor diagonal axial charges in Ref. [3], and for flavor diagonal tensor charges in Ref. [4].

High-statistics data were generated cost-effectively using the truncated solver with bias correction method (TSM) [5]. We also used the coherent source method to construct sequential propagators with the insertion of a zero-momentum nucleon state at the sink time slice [6].

The excited-state contamination (ESC) in the 3-point correlation functions was analyzed by generating, on each ensemble, data at 3-6 values of the source-sink separation $\tau$. Fits to extract the ground state ME were then made keeping up to three states in the spectral decomposition to data at a large set of values of the operator insertion time $t$ and $\tau$. The choice of the number of states kept (1, 2 or 3$)$ depended on the statistical precision of the data and the size of the ESC. The amplitudes and masses of the ground and excited states used in these fits were obtained from 2-point functions keeping four states in fits using the spectral decomposition. The 2-state (one-state, i.e., constant) fits to extract the disconnected $g_{A}^{l}\left(g_{T}^{l}\right)$ are shown in Fig. 2.

All the isovector renormalization constants $Z_{\Gamma}$ have been determined non-pertubatively in the RI-sMOM scheme and then converted to $\overline{M S}$ at $2 \mathrm{GeV}$ using 2-loop perturbation theory. For the flavor diagonal axial and tensor charges, we assumed that the difference between $Z^{\text {isovector }}$ and $Z^{\text {isoscalar, }}$, which is zero at 2-loops [7], is small and negligible non-perturbatively.

To obtain estimates of the renormalized charges in the continuum limit $(a \rightarrow 0)$, physical pion mass $\left(M_{\pi^{0}}=135 \mathrm{MeV}\right)$ and in the infinite volume limit $(L \rightarrow \infty)$, we used a simultaneous chiralcontinuum-finite-volume (CCFV) fit ansatz with leading order corrections:

$$
g_{A, S, T}\left(a, M_{\pi}, L\right)=c_{1}+c_{2} a+c_{3} M_{\pi}^{2}+c_{4} M_{\pi}^{2} e^{-M_{\pi} L} .
$$

For our clover-on-HISC calculation, the leading discretization correction is $O(a)$ since the clover action and operators are not fully $O(a)$ improved. The CCFV fits for the isovector charges using data on 11 ensembles are shown in Fig. 1. The disconnected contributions are small in magnitude, have larger statistical errors and were analyzed on fewer ensembles, 6 for light quarks and 7 for the strange. The chiral-continuum extrapolation (neglecting the finite volume corrections as they were small in the connected contributions) for disconnected $g_{A}^{l, s}$ and $g_{T}^{l, s}$ is shown in Fig. 3.

\section{Results}

Using the CCFV fit ansatz given in Eq. (1.1), the continuum limit results for the isovector charges of the proton, at $M_{\pi}=135 \mathrm{MeV}$ and in the $\overline{M S}$ scheme at $2 \mathrm{GeV}$, are

$$
g_{A}^{u-d}=1.218(25)(30) ; \quad g_{S}^{u-d}=1.022(80)(60), \quad g_{T}^{u-d}=0.989(32)(10) .
$$

The results for the flavor diagonal axial and tensor charges, in the $\overline{M S}$ scheme at $2 \mathrm{GeV}$, are:

$g_{A}^{u} \equiv \Delta u \equiv\langle 1\rangle_{\Delta u^{+}}=0.777(25)(30), g_{A}^{d} \equiv \Delta d \equiv\langle 1\rangle_{\Delta d^{+}}=-0.438(18)(30), g_{A}^{s} \equiv \Delta s=-0.053(8)$. 

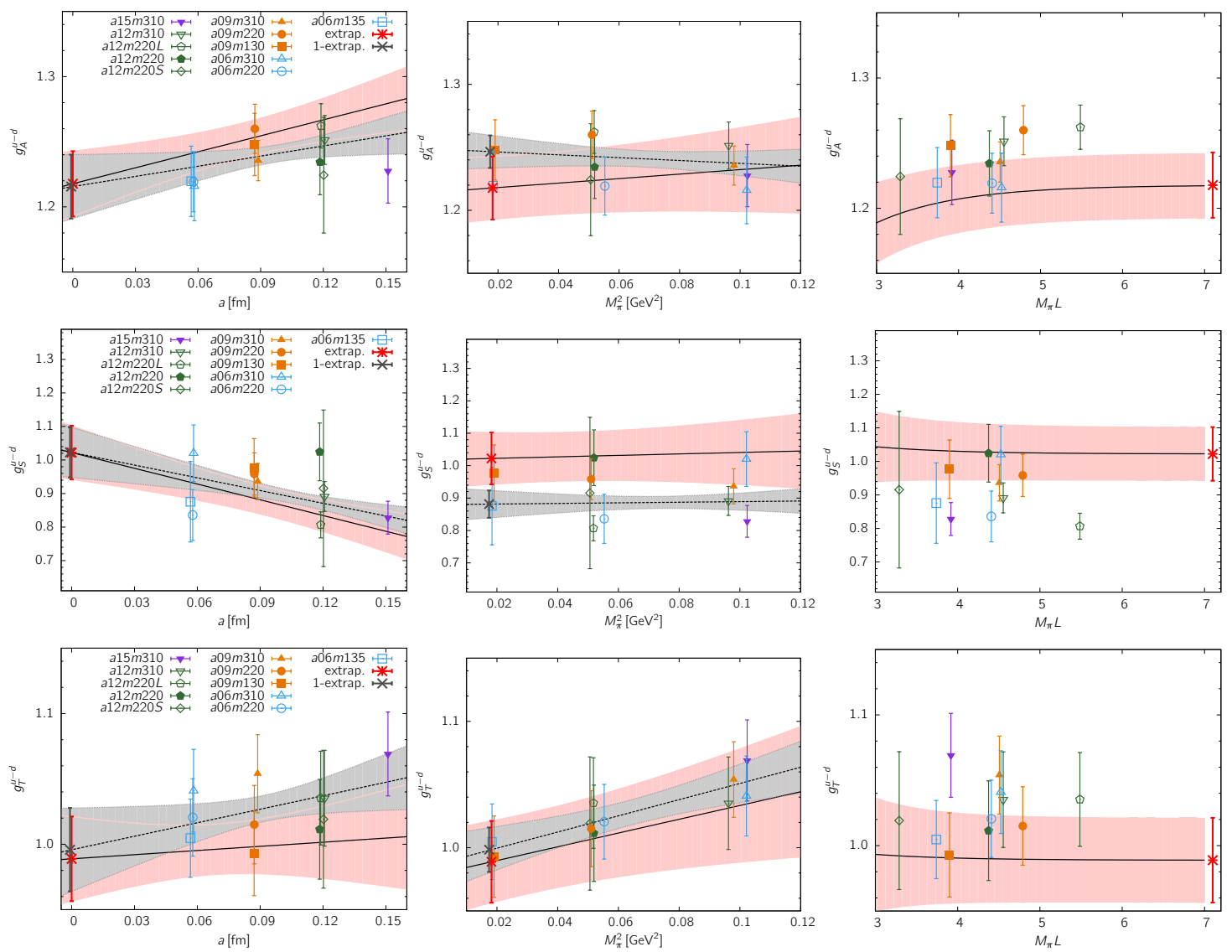

Figure 1: The 11-point CCFV fit using Eq. (1.1) to the data for the renormalized isovector charges $g_{A}^{u-d}$, $g_{S}^{u-d}$, and $g_{T}^{u-d}$ in the $\overline{\mathrm{MS}}$ scheme at $2 \mathrm{GeV}$. The result of the simultaneous extrapolation to the physical point at $a \rightarrow 0, M_{\pi} \rightarrow M_{\pi^{0}}^{\text {phys }}=135 \mathrm{MeV}$ and $M_{\pi} L \rightarrow \infty$ are marked by a red star. The pink error band in each panel is the result of the simultaneous fit but shown as a function of a single variable. The overlay in the left (middle) panels with the dashed line within the grey band is the fit to the data versus $a\left(M_{\pi}^{2}\right)$, i.e., neglecting dependence on the other two variables. The symbols used to plot the data are defined in the left panels.

$$
g_{T}^{u}=0.784(28)(10), \quad g_{T}^{d}=-0.204(11)(10), \quad g_{T}^{s}=-0.0027(16) .
$$

The second error accounts for the uncertainty due to the chiral-continuum ansatz, Eq. (1.1) .

\section{Phenomenology}

The isovector axial charge is a benchmark quantity for the calculations of nucleon matrix elements since it is known with high accuracy experimentally. Our result, $g_{A}^{u-d}=1.218(25)(30)$, is about $5 \%$ or $1.5 \sigma$ below the latest experimental value $g_{A}^{u-d}=1.2772(20)$ [8] and the recent CalLat result, $g_{A}^{u-d}=1.271$ (13) [9]. In Ref. [2], we show that the difference from the CalLat result comes from differences in the chiral and continuum extrapolation. CalLat has not simulated the three HISQ ensembles at $a \approx 0.57 \mathrm{fm}$, which pull down our result (see Fig. 1). Also, their data on the two physical mass ensembles have large errors and do not contribute significantly to the fits, i.e., their estimate is essentially based on fits to data with $M_{\pi} \geq 220 \mathrm{MeV}$. Our conclusion is that 

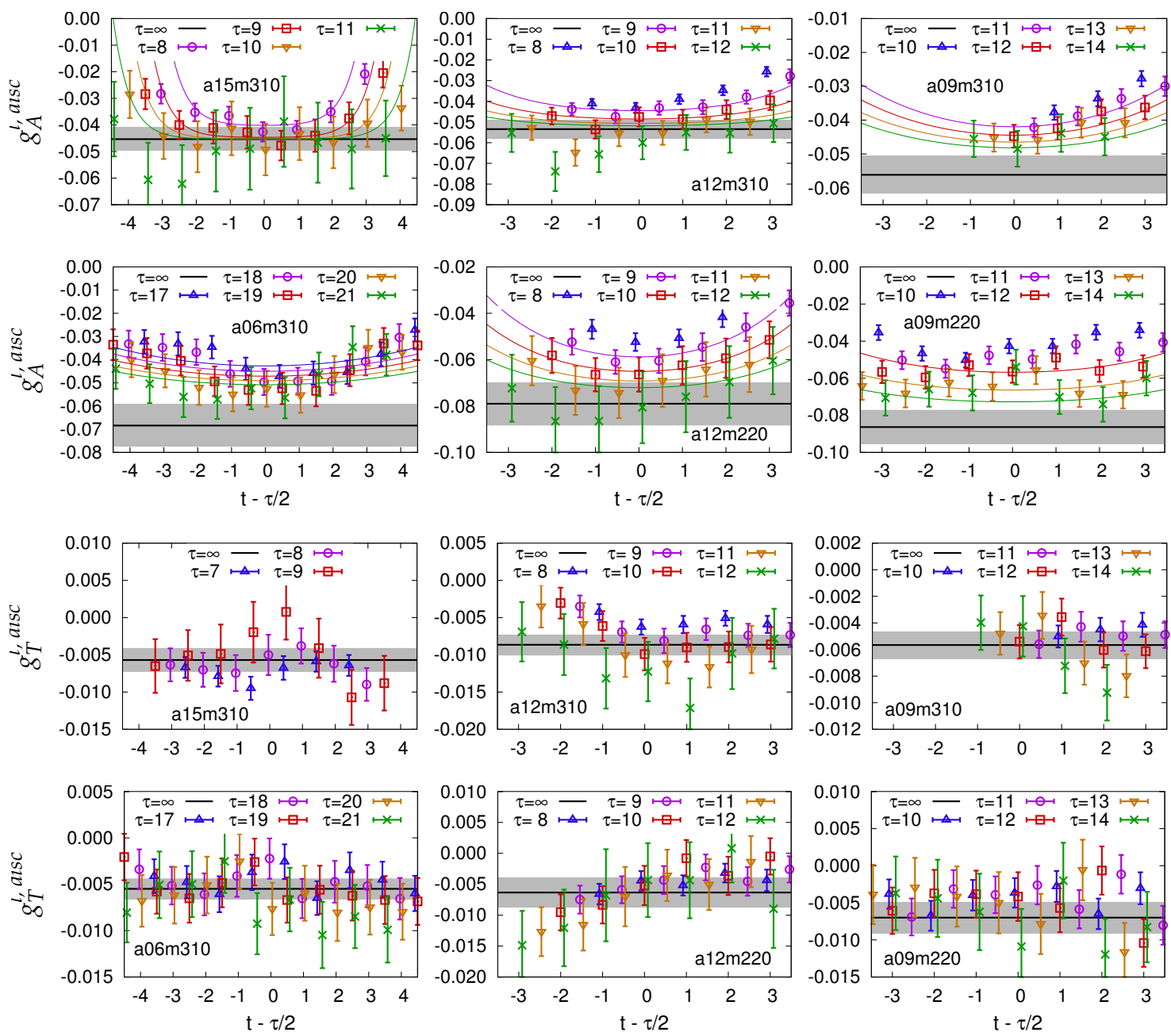

Figure 2: The data and excited-state fits for the light quark disconnected contribution to the bare $g_{A}^{l, s d i s c}$ (top 2 rows) and $g_{A}^{l, s d i s c}$ (bottom 2 rows) The grey error band and the solid line within it is the $\tau \rightarrow \infty$ estimate obtained using the 2-state (constant) fit to $g_{A}^{l, \text { disc }}\left(g_{T}^{l, \text { disc }}\right)$ data at different $t$ and $\tau$. The result of the fit for each individual $\tau$ is shown by a solid line in the same color as the data points.

more extensive calculations (larger range of $a$, more high statistics physical mass ensembles and different actions) are needed to understand and quantify the systematics at the percent level.

The scalar, $g_{S}^{u-d}$, and tensor, $g_{T}^{u-d}$, charges, combined with low energy neutron decay experiments, provide bounds on novel scalar and tensor interactions that can arise in BSM models at the TeV scale [10]. Our results, given in Eq. (2.1), have reached the $10 \%$ accuracy needed to complement measurements of helicity flip parameters $b$ and $B$ in neutron decay at the $10^{-3}$ level [10]. In Fig. 4, we compare present and future bounds from low-energy and LHC experiments, and find that, to match future LHC bounds, low energy experiments need to achieve better than $10^{-3}$ accuracy.

The quark spin contribution to the spin of the proton is given by $\sum_{q=u, d, s}\left(\frac{1}{2} \Delta q\right)=0.143(31)(36)$ using the results given in Eq. (2.2). This estimate is obtained without model assumptions and is in good agreement with the recent COMPASS analysis $0.13<\frac{1}{2} \Delta \Sigma<0.18$ [13].

The contribution of the quark electric dipole moment operator for each quark flavor to the 

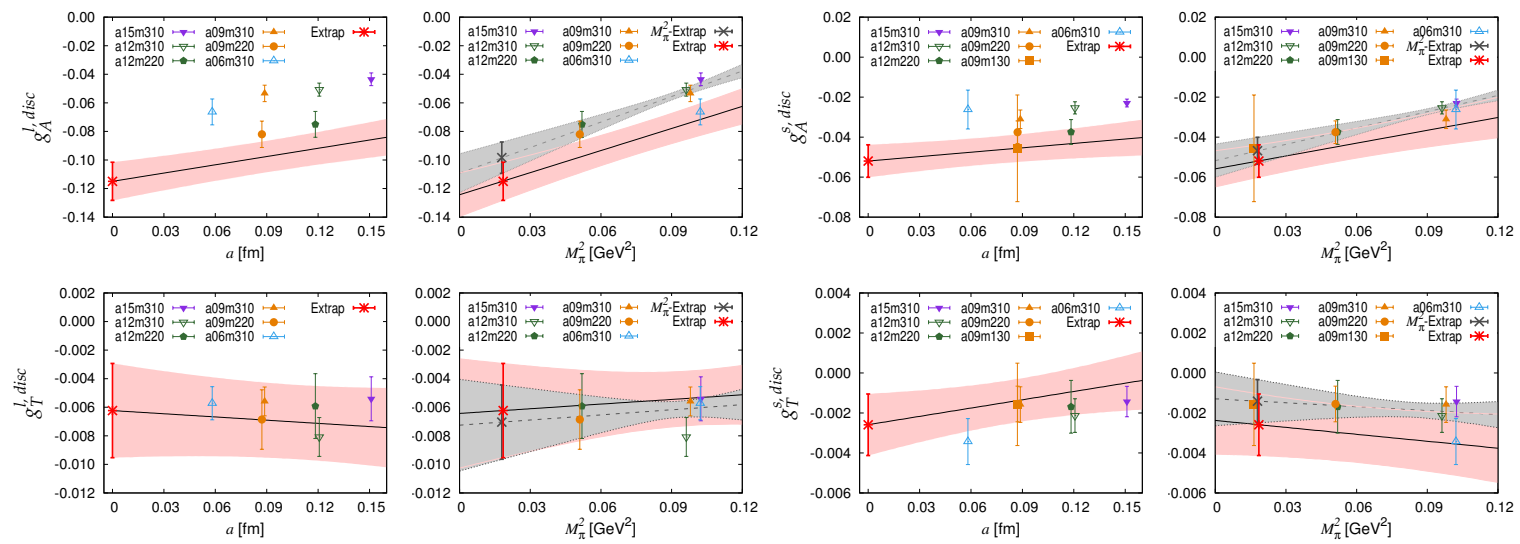

Figure 3: The extrapolation of the renormalized disconnected $g_{A}^{l, s}$ (top row) and $g_{T}^{l, s}$ data using the chiral-continuum ansatz given in Eq. (1.1). In each panel, the pink band shows the result of the simultaneous fit plotted versus a single variable with the other variable set to its physical value. The result at the physical point, $M_{\pi}=135 \mathrm{MeV}$ and $a=0$, is marked with a red star. The grey band shows the fit versus only $M_{\pi}$, i.e., ignoring the dependence on $a$. It highlights the need for a simultaneous fit in both $a$ and $M_{\pi}$.
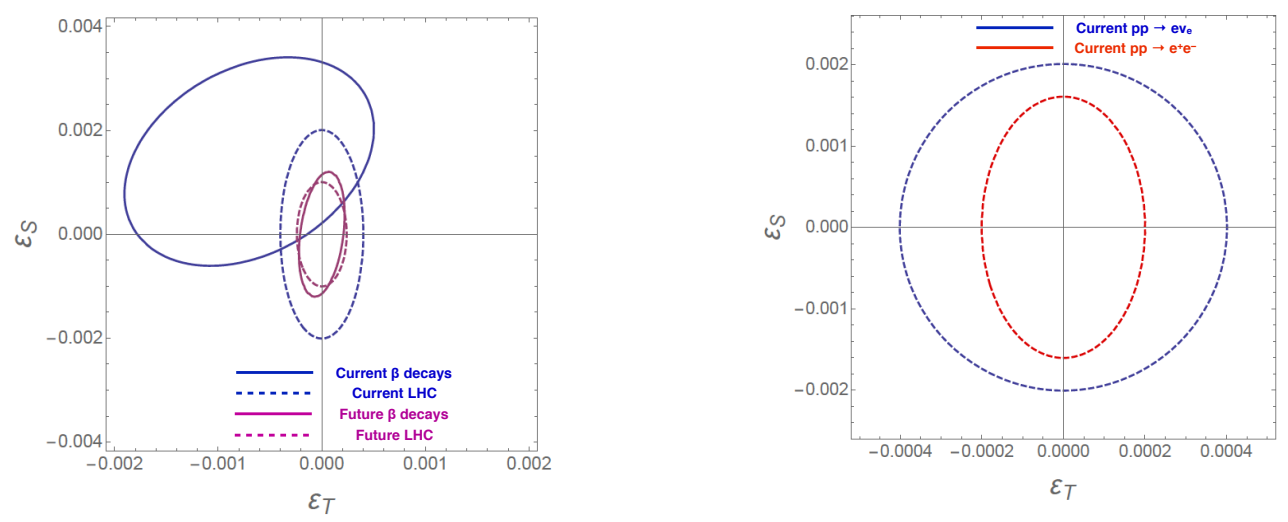

Figure 4: Current and projected $90 \%$ C.L. constraints on $\varepsilon_{S}$ and $\varepsilon_{T}$ defined at $2 \mathrm{GeV}$ in the $\overline{M S}$ scheme. (Left) The beta-decay constraints are obtained from the recent review article Ref. [11]. The current and future LHC bounds are obtained from the analysis of the $p p \rightarrow e+M E T+X$. We have used the ATLAS results [12], at $\sqrt{s}=13 \mathrm{TeV}$ and integrated luminosity of $36 \mathrm{fb}^{-1}$. We find that the strongest bound comes from the cumulative distribution with a cut on the transverse mass at $2 \mathrm{TeV}$. The projected future LHC bounds are obtained by assuming that no events are observed at transverse mass greater than $3 \mathrm{TeV}$ with an integrated luminosity of $300 \mathrm{fb}^{-1}$. (Right) Comparison of current LHC bounds from $p p \rightarrow e+M E T+X$ versus $p p \rightarrow e^{+} e^{-}+X$.

neutron electric dipole moment is given by

$$
d_{n}=d_{u}^{\gamma} g_{T}^{u}+d_{u}^{\gamma} g_{T}^{u}+d_{u}^{\gamma} g_{T}^{u}+\ldots,
$$

where $g_{T}^{u, d, s}$ are the flavor diagonal tensor charges given in Eq. (2.3) with $u \leftrightarrow d$ interchange for neutrons. Using these and the current experimental bound on the nEDM, $2.9 \times 10^{-26} e \mathrm{~cm}$ [14], we can constrain the quark EDMs $d_{u, d, s}^{\gamma}$ as shown in Fig. 5 (left). Conversely, given the values of $d_{u, d, s}^{\gamma}$ for a given BSM model, one can use our results for $g_{T}^{u, d, s}$ to bound $d_{n}$ provided quark 
EDMs are the dominant CP-violating operators that arise in BSM theories since each CP-violating operator contributes to $d_{n}$ with terms analogous to Eq. (3.1). It turns out that in the "split SUSY" model $[15,16,17]$, the fermion EDM operators provide the dominant BSM source of CP violation [18]. For this model, the allowed contour plots for $d_{n} / d_{e}$ in the gaugino $\left(M_{2}\right)$ and Higgsino $(\mu)$ mass parameter plane with the range $500 \mathrm{GeV}$ to $10 \mathrm{TeV}$ are shown in Fig. 5 (right). For this analysis, we have followed Ref. [19] and set $\tan \beta=1$. In particular, our results, assuming maxi-

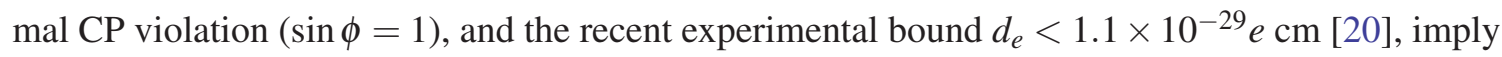
the split-SUSY upper bound $d_{n}<4.1 \times 10^{-29} e \mathrm{~cm}$. This limit is falsifiable by the next generation nEDM experiments.
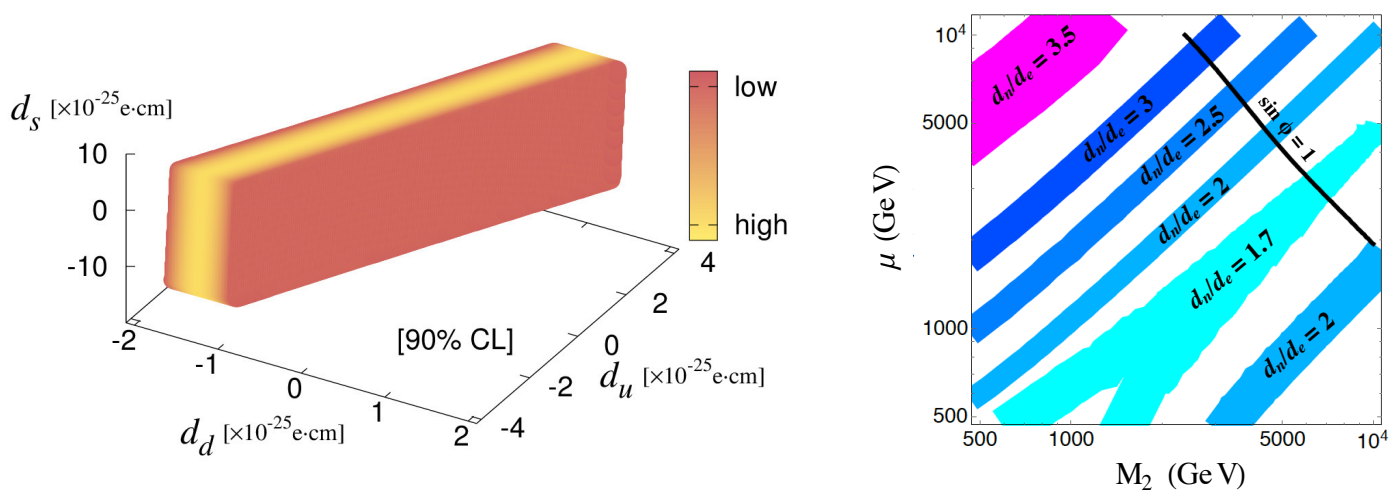

Figure 5: (Left) Constraints on the BSM couplings of the CP-violating quark EDM operator using the current experimental bound on the $\operatorname{nEDM}\left(2.9 \times 10^{-26} e \mathrm{~cm}[14]\right)$ and assuming that only these couplings contribute. The strongest constraint is a strip in $d_{u}$ and $d_{d}$, i.e., representing the thickness of the slab, with high (low) corresponding to a p-value $=1(0.1)$. (Right) Regions in $M_{2}-\mu$ plane corresponding to various values of $d_{n} / d_{e}$ in split SUSY, obtained by varying $g_{T}^{u, d, s}$ within our estimated uncertainties. In the bands of constant $d_{n} / d_{e}$, the values of both $d_{n}$ and $d_{e}$ decrease as $\mu$ and $M_{2}$ increase. Using $d_{e} \leq 1.1 \times 10^{-29} \mathrm{e}$ $\mathrm{cm}[20]$ and assuming maximal $\mathrm{CP}$ violation $(\sin \phi=1)$, the allowed region lies above the solid black line. For $\mu, M_{2}>500 \mathrm{GeV}$, maximizing the ratio $d_{n} / d_{e}$ along this line gives the upper bound $d_{n}<4.1 \times 10^{-29} \mathrm{e}$ $\mathrm{cm}$ at $d_{n} / d_{e}=3.71$.

\section{Acknowledgments}

We thank the MILC collaboration for sharing the $2+1+1$-flavor HISQ ensembles generated by them. We gratefully acknowledge the computing facilities at and resources provided by NERSC, Oak Ridge OLCF, USQCD and LANL Institutional Computing.

\section{References}

[1] MILC collaboration, A. Bazavov et al., Lattice QCD ensembles with four flavors of highly improved staggered quarks, Phys. Rev. D87 (2013) 054505 [1212.4768].

[2] R. Gupta, Y.-C. Jang, B. Yoon, H.-W. Lin, V. Cirigliano and T. Bhattacharya, Isovector Charges of the Nucleon from 2+1+1-flavor Lattice QCD, Phys. Rev. D98 (2018) 034503 [1806. 0900 6]. 
[3] H.-W. Lin, R. Gupta, B. Yoon, Y.-C. Jang and T. Bhattacharya, Quark contribution to the proton spin from 2+1+1-flavor lattice QCD, Phys. Rev. D98 (2018) 094512 [1806. 10604 ].

[4] R. Gupta, B. Yoon, T. Bhattacharya, V. Cirigliano, Y.-C. Jang and H.-W. Lin, Flavor diagonal tensor charges of the nucleon from (2+1+1)-flavor lattice QCD, Phys. Rev. D98 (2018) 091501 [1808.07597].

[5] G. S. Bali, S. Collins and A. Schafer, Effective noise reduction techniques for disconnected loops in Lattice QCD, Comput.Phys.Commun. 181 (2010) 1570 [0910.3970].

[6] B. Yoon et al., Controlling Excited-State Contamination in Nucleon Matrix Elements, Phys. Rev. D93 (2016) 114506 [1602.07737].

[7] M. Constantinou, M. Hadjiantonis, H. Panagopoulos and G. Spanoudes, Singlet versus nonsinglet perturbative renormalization of fermion bilinears, Phys. Rev. D94 (2016) 114513 [1610 06744 ].

[8] UCNA collaboration, M. A. P. Brown et al., New result for the neutron $\beta$-asymmetry parameter $A_{0}$ from UCNA, Phys. Rev. C97 (2018) 035505 [1712.00884].

[9] C. C. Chang et al., A per-cent-level determination of the nucleon axial coupling from quantum chromodynamics, Nature 558 (2018) 91 [1805.12130].

[10] T. Bhattacharya, V. Cirigliano, S. D. Cohen, A. Filipuzzi, M. Gonzalez-Alonso et al., Probing Novel Scalar and Tensor Interactions from (Ultra)Cold Neutrons to the LHC, Phys.Rev. D85 (2012) 054512 [1110.6448].

[11] M. GonzÃąlez-Alonso, O. Naviliat-Cuncic and N. Severijns, New physics searches in nuclear and neutron $\beta$ decay, 1803.08732 .

[12] ATLAS collaboration, M. Aaboud et al., Search for a new heavy gauge boson resonance decaying into a lepton and missing transverse momentum in $36 \mathrm{fb}^{-1}$ of pp collisions at $\sqrt{\mathrm{s}}=13 \mathrm{TeV}$ with the ATLAS experiment, Eur. Phys. J. C78 (2018) 401 [1706.04786].

[13] COMPASS collaboration, C. Adolph et al., The spin structure function $g_{1}^{\mathrm{p}}$ of the proton and a test of the Bjorken sum rule, Phys. Lett. B753 (2016) 18 [1503.08935].

[14] C. Baker, D. Doyle, P. Geltenbort, K. Green, M. van der Grinten et al., An Improved experimental limit on the electric dipole moment of the neutron, Phys.Rev.Lett. 97 (2006) 131801 [hep-ex/ 0602020 ].

[15] N. Arkani-Hamed and S. Dimopoulos, Supersymmetric unification without low energy supersymmetry and signatures for fine-tuning at the LHC, JHEP 0506 (2005) 073 [hep-th / 0405159 ].

[16] G. Giudice and A. Romanino, Split supersymmetry, Nucl.Phys. B699 (2004) 65 [hep-ph/0406088].

[17] N. Arkani-Hamed, S. Dimopoulos, G. Giudice and A. Romanino, Aspects of split supersymmetry, Nucl.Phys. B709 (2005) 3 [hep-ph / 0409232$].$

[18] T. Bhattacharya, V. Cirigliano, R. Gupta, H.-W. Lin and B. Yoon, Neutron Electric Dipole Moment and Tensor Charges from Lattice QCD, Phys. Rev. Lett. 115 (2015) 212002 [1506. 04196 ].

[19] G. Giudice and A. Romanino, Electric dipole moments in split supersymmetry, Phys.Lett. B634 (2006) 307 [hep-ph/ 0510197$].$

[20] ACME collaboration, V. Andreev et al., Improved limit on the electric dipole moment of the electron, Nature 562 (2018) 355. 